\title{
Commissioning and performance of the CMS calorimeter system with proton-proton collisions at the LHC
}

\author{
Philippe Gras* on behalf of the CMS Collaboration \\ CEA/IRFU - Saclay \\ E-mail: philippe.gras@email
}

On March 30th, 2010, the CMS experiment at the LHC saw the first p-p collisions at $7 \mathrm{TeV}$ center-of-mass energy. The calorimeter system of the CMS detector is made of a high precision homogeneous electromagnetic calorimeter covering the pseudorapidity acceptance $|\eta|<3$ and a sampling hadronic calorimeter covering the acceptance downto $|\eta|=5.2$. The design resolution is $0.5 \%$ at higher energy for the electromagnetic calorimeter and $100 \% / \sqrt{\mathrm{E}} \oplus 5 \%$ for the hadronic one. Precalibration of the calorimeters allowed starting in excellent conditions, with very good performance, and a good understanding of the detector showed by an accurate prediction of its reponse from the Monte-Carlo simulation.

35th International Conference of High Energy Physics

July 22-28, 2010

Paris, France

${ }^{*}$ Speaker. 
The Compact Muon Solenoid (CMS) experiment [1], on the Large Hadron Collider (LHC) [2] started to collect its first data with proton-proton collisions at $7 \mathrm{TeV}$ in the centre of mass on March 30th of the 2010 year. The diphoton mass resolution is essential for search of Higgs boson lighter than $130 \mathrm{GeV} / \mathrm{c}^{2}$, requiring an excellent resolution of the electromagnetic calorimeter. Supersymmetry searches requires a good missing transverse energy measurement, which demands calorimeters with a large hermetic coverage. In this letter we will present the CMS calorimeter system and its performance at start-up

\section{Description of the calorimeter system}

The CMS detector is equipped of a $12.5 \mathrm{~m}$ long and $6.3 \mathrm{~m}$ diameter $3.8 \mathrm{~T}$ superconducting solenoid. The calorimeters are placed inside this magnet.

The hadronic calorimeter (HCAL) is a sampling calorimeter which covers the pseudorapidity region $|\eta|<5$.2. It is completed outside the solenoid by a tail catcher in the barrel region $(|\eta|<$ 3 ), which uses the solenoid coil as absorber. The $3<|\eta|<5.2$ region is covered by a forward calorimeter, located $11.2 \mathrm{~m}$ from the interaction point. The part in the solenoid (barrel and endcap) uses brass as absorber and BiCron BC408 scintillator as active material. Light is collected with wavelength shift fibre. The total absorber thickness at $90^{\circ}$ is 5.82 interaction lengths. The readout granularity is $\eta=0.087 \times 0.087$ for $|\eta|<1.6$ and $\eta \approx 0.17 \times 0.17$ for $|\eta|>1.6$. The calorimeter in the forward region uses steel as absorber. The active part is made of quartz fibres, where light is produced by Čerenkov effect. The fibres run parallel to the beam. The usage of two fibre lengths allows separation of electrons and photons from hadrons based on their shower profile. More than 99.75\% channel of the hadronic calorimeter are operational.

The electromagnetic calorimeter (ECAL) is a homogeneous calorimeter. It uses lead tungstate crystals, chosen for the short radiation length $(0.89 \mathrm{~cm})$, the small Molière radius $(2 \mathrm{~cm})$ and the fast scintillation. In the barrel region $(|\eta|<1.479)$, the granularity is $\eta \times \phi=0.0174 \times 0.0174$ and the depth is 26 radiation length. In the endcap region $(1.479<|\eta|<3.0)$ the front face of the crystals sizes $29.6 \mathrm{~mm} \times 29.6 \mathrm{~mm}$ providing a granularity from $0.021 \times 0.021$ to $0.050 \times 0.050$ depending on pseudorapidity, the crystal depth covers 25 radiation length. In the endcap region A preshower detector, made of two lead layers interleaved with two silicon strip layers, is used to enhance the $\pi^{0}-\gamma$ separation. The target resolution of the electromagnetic calorimeter is $0.5 \%$ at high energy. The running conditions are extremely stable, the temperature fluctuations are limited to $0.003^{\circ} \mathrm{C} \mathrm{RMS}$ in the barrel, $0.015^{\circ} \mathrm{C}$ in the endcap meeting the stringent requirements imposed by the resolution target. The response to laser light, used to monitor the crystal transparency which varies due to irradiation [3], presents a stability better than $0.02 \%$. The number of dead channels is very small, less than $1 \%[4]$.

\section{Calorimeter system performance at start-up}

Both calorimeters were precalibrated before their installation in CMS. The 10 year long campaign of calibration and test beam allowed starting in excellent conditions and with a very good understanding of the apparatus. 
The design goal of the ECAL channel-to-channel calibration is $0.3 \%$ and will be achieved using electromagnetic decays of $\mathrm{W}$ and $\mathrm{Z}$ bosons. The off-site precalibration conducted using cosmic muons provided a precision at start-up of $1.5 \%$ to $2.2 \%$ in the barrel depending on the pseudorapidity for every channel and $5 \%$ in the endcap. Nine of the 36 modules constituting the barrel were independently precalibrated with an electron beam at a precision better than $0.5 \%$ [5]. A strategy was developed to select $\pi^{0}$ and $\eta$ mesons with a dedicated trigger stream and use their decay in two photons for calibration in the limited luminosity startup conditions (see Figure 1). With the first $250 \mathrm{nb}^{-1}$ the $\pi^{0}$ in-situ channel-to-channel calibration, combined with a $\phi$-symmetry calibration, reached a precision of

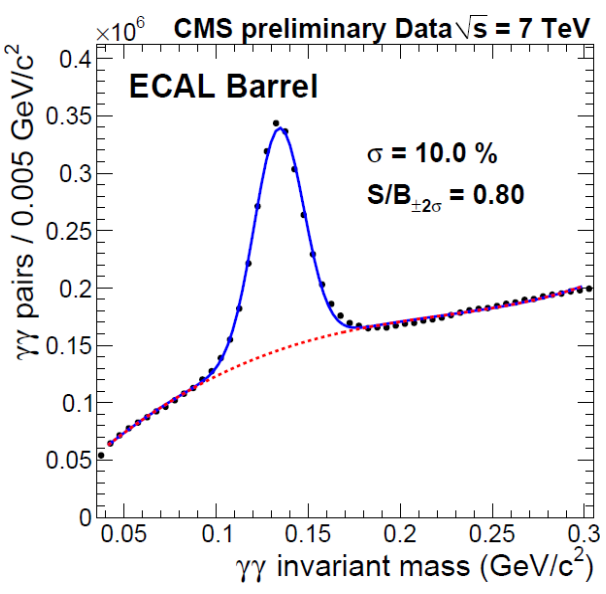

Figure 1: $\pi^{0}$ invariant mass reconstructed from photon pairs accepted by off-line selection obtained with $0.31 \mathrm{nb}^{-1}$. $0.6 \%$ in the central part of the barrel $(|\eta|<0.785)[6]$.

This measurement asserted the validity of precalibration obtained off-site for its in-situ use. Its accuracy is limited by statistical uncertainties and is getting improved while acquiring new data and is expected to reach $0.5 \%$ in the barrel and 1 to $2 \%$ in the endcaps. Preshower was calibrated with a precision of $2.2 \%$ already achieving the design goal. The first data showed a very good matching with the Monte-Carlo simulation, without any tuning, as illustrated on Figure 2, demonstrating a good understanding of the detector.

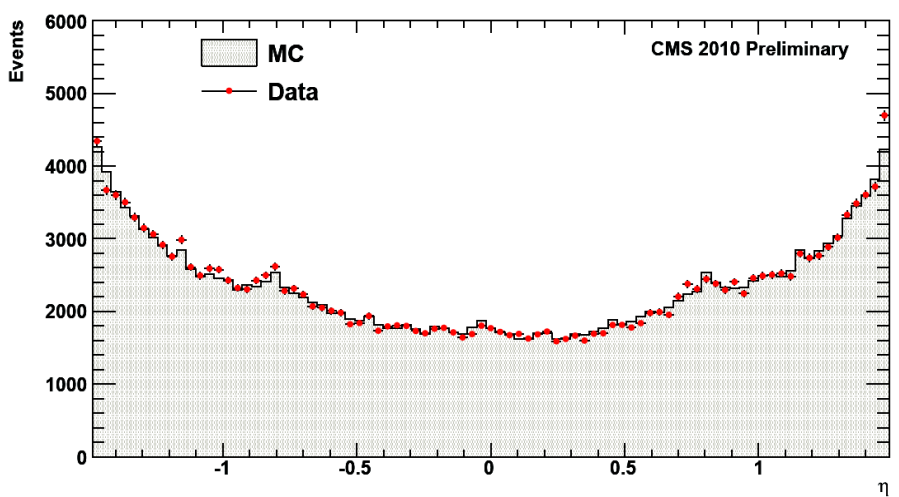

Figure 2: Pseudorapidity distributions of the ECAL barrel channel with the highest reconstructed energy in $7 \mathrm{TeV}$ minimum bias collision events.
The precalibration of $\mathrm{HCAL}$ combined test beam calibration, in-situ channel-to-channel calibration with a ${ }^{60} \mathrm{Co}$ source, in-situ cosmic ray events and "splash" events (single beam shots sent to closed collimator near CMS experiment resulting in a large flux of muon traversing the detector). The channel-to-channel precalibration has a precision of $5 \%$ to $12 \%$ for $85 \%$ of the barrel channels, $10 \%$ for endcap channels, $12 \%$ for the forward detector and $5 \%$ in the tail catcher [7].

The precalibration and the good understanding of the calorimeter response, allowed a start up with an energy scale calibration obtained from Monte-Carlo with a fair precision of $10 \%+2 \% \cdot|\eta|$ for jet reconstruction using only the calorimeter information and $5 \%+2 \% \cdot|\eta|$ for algorithms combining calorimeter and tracker information. The first $71 \mathrm{nb}^{-1}$ of data were used to assert the validity of this precision. They support the quoted uncertainties as conservative numbers [8]. Figure 3 shows the resolution of the jet transverse momentum as measured with the dijet asymmetry 
method [9]. Data driven methods were developed to calibrate the jet energy and will be used with the coming data. They will allow achieving the design goal precision, $5 \%$ on absolute scale, $0.5 \%$ $(2 \%)$ on relative scale for barrel with an integrated luminosity of $10 \mathrm{pb}^{-1}$.

The understanding of the detector response is even more important for missing energy measurement. The resolution of transverse components $E_{x, y}^{m i s s}$ of missing energy measurement for dijet events is shown on figure 4 [11]. The resolution is very good, less than $11 \mathrm{GeV}$ with the particle flow algorithm [10] on the whole $\sum E_{\mathrm{T}}$ range from $100 \mathrm{GeV}$ to $350 \mathrm{GeV}$. This algorithm combines all the subdetector information to reconstruct all particles produced by the collision. Its performance is compared to calorimetric-based method, showing a very nice enhancement. The good agreement with the Monte Carlo for both algorithms asserts the understanding of the detector response.

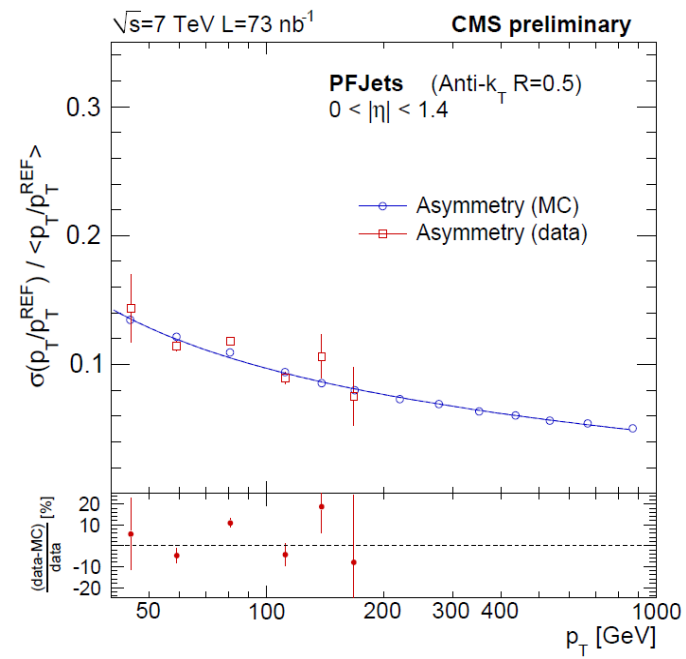

Figure 3: Jet resolution in pseudorapidity region $0<|\eta|<1.4$ determined with the asymmetry method from QCD simulation and compared with the result from data using the same procedure. Jets are reconstructed with the particle flow algorithm.

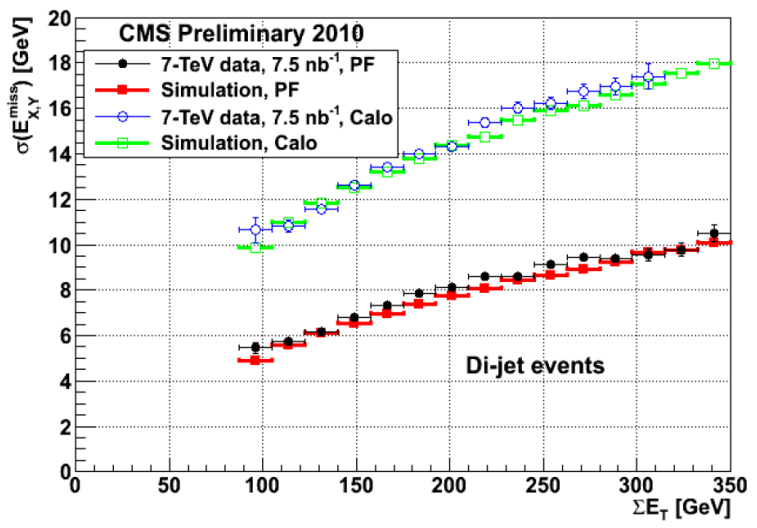

Figure 4: Resolution of $E_{\mathrm{x}, \mathrm{y}}^{\mathrm{miss}}$ for particle-based (solid symbols) and calorimeter-based (hollow symbols) reconstruction in data (dots) and simulation (squares), as a function of the particle based $\sum \mathrm{E}_{\mathrm{T}}$, for dijet events.

The CMS calorimeter system showed an excellent performance since the very beginning of the data taking, an essential ingredient for the first physics analysis presented in this conference. The commissioning is essentially finished. It has fully exploited the limited amount of available data. The detector calibration is advancing very fast with the LHC luminosity ramp-up.

\section{References}

[1] The CMS Collaboration, 2008 JINST 3 S08004

[2] Lyndon Evans and Philip Bryant, 2008 JINST 3 S08001

[3] M. Anfreville at al., NIM A, 594, pp 292-320 (2008). Monitoring the stability of the CMS electromagnetic calorimeter, F. Ferri, presented at Calor 2010, proceedings to be published in J. Phys.: Conf.

[4] The CMS Collaboration, CMS NOTE 2010/12 (2010) 
[5] The CMS Electromagnetic Calorimeter Group, 2008 JINST 3 P10007

[6] The CMS Collaboration, PAS EGM-2010-003 (2010)

[7] The CMS-HCAL Collaboration, Eur. Phys. J. C 55, 159-171 (2008) and 2010 JINST 5 T03012. The CMS HCAL/ECAL Collaboratiob, Eur. Phys. J. C (2009) 60: 359Ü373

[8] The CMS Collaboration, CMS PAS JME-10-003 (2010)

[9] The D0 Collaboration, Phys.Rev.D64 032003 (2000). The CMS Collaboration, CMS PAS JME-09-007 (2009).

[10] The CMS Collaboration, CMS PAS PFT-10-001 (2010)

[11] The CMS Collaboration, CMS PAS PFT-10-002 (2010), CMS PAS JME-10-004 (2010) and CMS PAS JME-10-005 (2010) 